
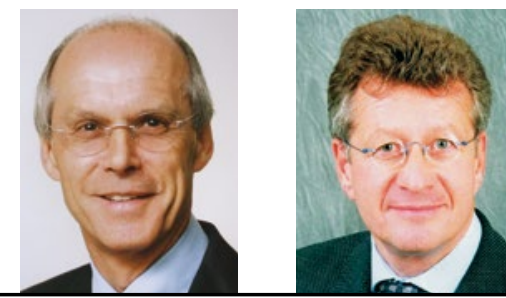

Dr. med. P. Stiefelhagen

Internist,

Hachenburg

\title{
Was tun bei ständigen schweren Infekten?
}

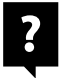 Dr. Claudia Petroni, Brixen/Südtirol: Meine 35-jährige Patientin durchlebt alle paar Wochen bis Monate teils schwere Infekte, im Sommer etwa eine schwere Pneumonie mit stationärem Aufenthalt und Rezidiv innerhalb von zwei Wochen. Im Spital wurde angedeutet, man sollte das Immunsystem genauer unter die Lupe nehmen, da u. a. ein Mangel an den Komplementfaktoren C3 und C4 im Blut vorliege. Was könnte ich diesbezüglich tun?}

\section{Tab. 1 Fünf Warnzeichen für primäre Immundefekte}

- Pathologische Infektanfälligkeit nach dem ELVIS-Schema: Erreger, Lokalisation, Verlauf, Intensität, Summe

- Immundysregulation nach dem GARFIELD-Schema: Granulome, Autoimmunität, rezidivierendes Fieber, ungewöhnliche Ekzeme, Lymphproliferation, chronische Darmentzündung

- Gedeihstörung (Kinder) bzw. Gewichtsverlust, meist mit Diarrhö (Erwachsene)

- Auffällige Familienanamnese

- Labor: Lymphopenie, Neutropenie, Hypogammaglobulinämie

Quelle: Leitlinie "Diagnostik von primären Immundefekten", AWMF-Registernummer 027/050

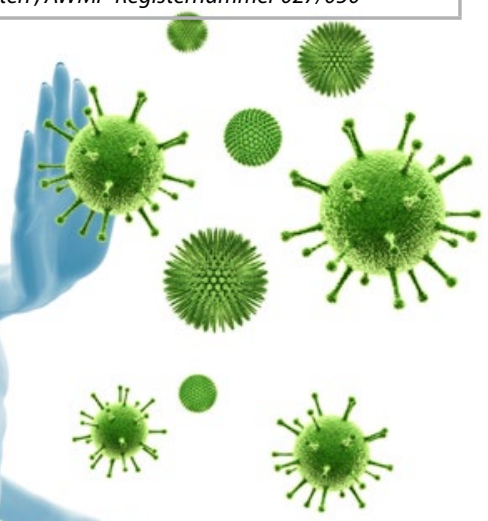

MMW-Experte Stiefelhagen: Bei Verdacht auf einen primären oder sekundären Immundefekt sind ein Differenzialblutbild, eine quantitative Analyse der B-Lymphozyten-Subgruppen (Lymphozytenphänotypisierung), eine Serumeiweißelekrophorese und eine quantitative Bestimmung der Immunglobuline (IgG, IgA, IgM, IgE, IgG-Subklassen) angezeigt. Als Globaltest zur Untersuchung des klassischen Komplementwegs dient $\mathrm{CH} 50$.

Bei der Planung, Durchführung und Bewertung der erhobenen Befunde empfiehlt sich aber die enge Zusammenarbeit mit einem in der Diagnostik und Behandlung von Immundefekten erfahrenen Arzt. Bei einer derartigen Schrotflintendiagnostik können sich etliche grenzwertige, schwer zu interpretierende Befunde ergeben.

! MMW-Experte Füeßl: Eine pathologische Infekthäufigkeit, insbesondere im Erwachsenenalter, ist unscharf definiert; größere Studien fehlen. Zeichen einer pathologischen Infektanfälligkeit können Infektionen durch op- portunistische Erreger (Pneumocystis, Candida, Kryptosporidien, Mikrosporidien, atypische Mykobakterien) sein, die bei immunkompetenten Personen nur selten zu schweren Erkrankungen führen. Auch schwere, rezidivierende Infektionen mit "gewöhnlichen“ Erregern wie Pneumokokken oder Herpessimplex-Viren können auf einen primären Immundefekt hinweisen.

Für eine systemische Abwehrschwäche sprechen auch vorwiegend polytope Infektionen oder atypische Lokalisationen, ein protrahierter Verlauf oder ein unzureichendes Ansprechen auf gängige Antibiotika. Eine schwedische Arbeitsgruppe hat dazu 2009 fünf Warnzeichen zusammengefasst (Tab. 1).

Bei Verdacht auf einen primären Immundefekt sollte eine Stufendiagnostik erfolgen. Als Basisdiagnostik dienen die Bestimmung der Immunglobuline (IgA, IgM, IgG, IgE) und ein Blutbild mit Differenzierung. Hilfreich kann auch eine Bestimmung von Impf-Antikörpern sein, wobei man für die Interpretation wissen sollte, welche Impfungen der Patient in welchem Alter erhalten hat.

Weitere Klarheit können Lymphozyten-Phänotypisierung, Eiweißelektrophorese und Komplementbestimmung schaffen. Spätestens ab hier sollte sich der Hausarzt allerdings mit einem in der Immundefektdiagnostik erfahrenen Arzt in Verbindung setzen. 